\title{
CONSEQUÊNCIAS DO USO DE DROGAS DURANTE A GRAVIDEZ
}

\author{
Jair Alves Maia*, Leonardo Assunção Pereira**, Fernanda de Alcântara Menezes*** \\ * Mestrando em Enfermagem pela Faculdade de Ciências da Saúde Albert Einstein São Paulo (2015), Pós-Graduado em \\ Didática do Ensino Superior (2015), Pós-Graduado em Saúde Pública (2008), Graduado em Enfermagem (2005). E-MAIL: \\ jairalvesac@hotmail.com \\ ** Mestrandos em Enfermagem pela Faculdade de Ciências da Saúde Albert Einstein São Paulo (2015). \\ *** Mestranda em Enfermagem pela Faculdade de Ciências da Saúde Albert Einstein São Paulo (2015).
}

\section{Resumo}

O avanço no consumo de drogas licitas e ilícitas nos dias de hoje é considerado como problemas de saúde pública em todo o mundo e principalmente no Brasil. Este estudo objetivou descrever as consequências do uso de drogas durante a gravidez. O método utilizado foi um estudo observacional e quantitativo com abordagem descritiva. Concluímos que $(37 ; 2,05 \%)$ das grávidas entrevistadas faziam uso de etanol, (19; 1,00\%) eram fumantes, $(22 ; 1,22 \%)$ eram usuária da maconha, (17; 0,94\%) faziam uso de cocaína e $(47 ; 2,61 \%)$ eram usuárias de crack, colocando em risco o desenvolvimento do feto e de sua saúde.

Palavras-chave: Drogas; Gravidez; Gonseqüências; Atenção primaria.

\section{CONSEQUENCES OF DRUG USE DURING PREGNANCY}

\begin{abstract}
The increase in the consumption of licit and illicit drugs today is considered as a public health problem worldwide and especially in Brazil. This study aimed to describe the consequences of drug use during pregnancy. The method used was an observational and quantitative study with descriptive approach. We conclude that $(37 ; 2.05 \%)$ of the interviewed pregnant women were using ethanol $(19,1.00 \%)$ were smokers, $(22 ; 1.22 \%)$ were user of marijuana $(17,0.94 \%)$ were using cocaine and $(47 ; 2.61 \%)$ were crack users, jeopardizing the development of the fetus and your health.

Keywords: Drugs; Pregnancy; Consequences; Primary care.
\end{abstract}




\section{INTRODUÇÃO}

O avanço no consumo de drogas licitas e ilícitas nos dias de hoje são considerados como problemas de saúde pública em todo o mundo e principalmente no Brasil onde se concentra um grande número de favelas e comunidades onde a expectativas de vida é muito baixa e o tráfico acabam sendo a única forma que vários jovens encontra para sua sobrevivência e da família. Por isso se faz necessário a realização de pesquisa sobre as conseqüências do uso de drogas na população, que muitas vezes entra nesse mundo sem saber que é um caminho que na maioria das vezes não tem volta.

A realização da presente pesquisa justifica-se pela importância de conhecer amplamente as consequências do uso de drogas licitas e ilícitas durante a gravidez, para a mãe e para o recém-nascido e a importância do acompanhamento da atenção primária à saúde, a população exposta e acometida pelo uso de drogas que hoje se configura como um problema de saúde pública no Brasil e no mundo.

A problemática desta pesquisa foi delineada com a seguinte pergunta: Quais são as drogas mais consumidas pelas grávidas que foram atendidas nas consultas de pré-natal na unidade de saúde Claudia Vitorino?, este estudo objetivou descrever as consequências do uso de drogas durante a gravidez e as variáveis que foram estudadas foram hábito de fumar, uso de etanol, uso da maconha, uso da cocaína e crack durante a gravidez com ênfase nas consequências para a população e especialmente para o feto.

O tabaco é a segunda droga mais consumida entre a população jovem, devido às facilidades e estímulos para adquirir o produto e ao desconhecimento dos graves prejuízos causados à saúde dos fumantes, esse consumo do tabaco tornou-se o hábito de fumar um comportamento socialmente aceitável. A prova disso é que $90 \%$ dos fumantes começam a fumar antes dos 19 anos de idade. Enfrentar o problema do tabagismo feminino, é um desafio para a Saúde Pública no século XXI, sendo que é necessário entender o fenômeno globalmen- te e agir localmente, com estratégias inovadoras e mais adequadas. ${ }^{(1)}$

O uso constante e descontrolado do etanol pode comprometer seriamente o funcionamento do organismo, levando a consequências irreversíveis. Depois do consumo essa substancia, é digerido no estômago e absorvido no intestino e através da corrente sanguínea suas moléculas são levadas ao cérebro. O uso prolongado do etanol pode prejudicar todos os órgãos, principalmente o fígado, que é responsável pela destruição das substâncias tóxicas ingeridas ou produzidas pelo corpo durante a digestão. Dessa forma com o uso abusivo, o fígado sofre uma sobrecarga para metabolizá-lo. Além disso, o abuso do dessa substância pode acarretar graves inflamações como: gastrite, hepatite alcoólica, pancreatite e neurite. ${ }^{(2)}$

$O$ etanol consumido pela gestante atravessa a barreira placentária, fazendo com que o feto seja exposto às mesmas concentrações do sangue materno. Contudo, a exposição fetal é maior, devido ao metabolismo e eliminação serem mais lentos, isso faz com que o líquido amniótico permaneça impregnado de dessa substância. Além disso, o uso e o abuso do etanol durante a gravidez está relacionado ao aumento do número de abortos e a fatores que podem comprometer o parto como, risco de infecções, deslocamento prematuro de placenta, hipertonia uterina, parto prematuro e presença de mecônio no líquido amniótico, colocando em risco a vida do feto e causando complicações na vida do recém-nascido. (3)

A maconha provavelmente seja a droga ilícita de maior consumo durante a gestação. Dentre os efeitos do uso da maconha, estão incluídos: diminuição da memória, perda da inibição, sensação de relaxamento ou euforia, alterações de percepção do tempo e espaço. Além disso, podem ocorrer alterações em outros sistemas do organismo como, aumento da freqüência cardíaca, vasodilatação, hiperemia conjuntival e aumento do apetite. Sendo que os efeitos mais significativos inclui o desenvol- 
vimento cognitivo e emocional. Observam-se ainda coincidências entre o uso de maconha e o mau desenvolvimento do tubo neural do RN, além de possíveis anencefalias (4)

A cocaína no organismo materno provoca grave vasoconstricção e, por atravessar a barreira placentária, estende esse efeito maléfico ao feto. Grande parte da agressão ao concepto é resultado da ação da droga que, ao diminuir o fluxo sanguíneo para o útero, para a placenta e para o feto pode ocasiona abortamento espontâneo, trabalho de parto prematuro, deslocamento prematuro da placenta, crescimento intrauterino retardado e sofrimento fetal crônico grave. Além de propiciar essas anormalidades no desenvolvimento da gravidez, a cocaína é hoje considerada teratogênica já que se observa nas gestações de usuárias da droga, maior prevalência de malformações fetais principalmente as do trato geniturinário, do coração e dos vasos da base e da face. ${ }^{(5)}$

No inicio da década de 80, uma nova droga foi descoberta nos Estados Unidos, descrita na literatura cientifica americana como uma mistura a base de bicarbonato de sódio, a pasta básica de cloridrato de cocaína. Com o aquecimento desses produtos se produz uma substância em forma de pedra, que quando queimado se produz um vapor inalável e um ruído típico de estalo, motivo pelo qual é chamado de crack. Este age no SNC diretamente sobre os neurônios atuando principalmente no bloqueio de recaptação de dopamina e noradrenalina. Esses são excitatórios e provoca a estimulação do SNC, causando efeitos como, sensações de euforia, ansiedade estado do alerta, etc. $O$ resultado desses efeitos provocam o aumento da pressão arterial e da frequência cardíaca, favorecendo assim, o risco de convulsão, infarto e derrame cerebral.(${ }^{(6)}$

O crack, é absorvido pelos alvéolos pulmonares e chega rapidamente à corrente sanguínea. $\mathrm{O}$ efeito do crack no sistema nervoso central é quase imediato, em média de 8 a 15 segundos, por esta razão, a droga pode causar dependência mais rapidamente. Enquanto a cocaína em pó leva cerca 15 minutos para chegar ao cérebro e surgir o efeito. ${ }^{(7)}$
São vários os prejuízos causados pelo uso de crack, Além das dificuldades respiratórias, causada pela aspiração de partículas sólidas, seu efeito estimulante induz à perda de apetite, insônia e agitação motora, além disso, a dificuldade que usuário tem para alimentar-se pode levar à desnutrição, desidratação e gastrite. Podem ainda ser observados sintomas físicos como: rachadura labial, pela falta de ingestão de água e de salivação, cortes e queimaduras nos dedos das mãos e às vezes no nariz, estes são provocados pelo ato de quebrar e acender a pedra. ${ }^{(8)}$

É importante ressaltar que o cuidado com as gestantes dependente de álcool e de outras drogas é complexo e exige um preparo específico por parte dos enfermeiros. Os profissionais devem estar conscientes das características únicas de cada usuária. O principal obstáculo para o tratamento das mulheres dependentes, em geral, é o preconceito que sofrem por parte da própria comunidade. ${ }^{(9)}$

Nas grávidas, esse preconceito se multiplica, tornando quase impossível um pedido de ajuda e como conseqüência, essas gestantes raramente fazem acompanhamento pré-natal e, quando fazem, ocultam o uso de drogas. Por outro lado, o período gestacional é um período facilitador de sensibilização ao tratamento, por isso se houver preparo por parte da equipe de saúde, é exatamente nessa fase que se consegue uma abstinência completa e duradoura de todas as drogas, tendo em vista que a maioria das mães não querem prejudicar o bebê. ${ }^{(10)}$

As ações de prevenção devem ser direcionadas as comunidades em situação de risco, com problemas decorrentes do uso de drogas. Dentre muitos fatores de risco relacionados o abuso de drogas, deve-se levar em consideração as precárias condições de moradia, presença de tráfico de drogas e ausência de perspectivas de trabalho. ${ }^{(11)}$

Além disso, é importante ressaltar que a família tem um papel essencial na prevenção do uso de drogas, por tanto é na relação direta com a comunidade, que se encontram os problemas e onde os profissionais das áreas de saúde e de educação poderão investir em ações e diretrizes de maneira preventiva, facilitando o acesso à educação, à 
saúde e a qualidade de vida dessas usuárias bem como dos seus familiares. ${ }^{(12)}$

As consultas de pré-natal são de fundamental importância, bem como, as orientações na prevenção do uso de drogas na gestação e no puerpério, estas orientações devem continuar, a fim de conscientizar principalmente as gestantes dos perigos e consequências da droga durante a gravidez e no período puerperal.(13)

Contudo, os profissionais da saúde principalmente os da estratégia saúde da família, por terem a família diariamente e diretamente participando das ações planejadas pela unidade, devem procurar nesse contexto, a construção de estratégias para auxiliar na prevenção, promoção, assistência e tratamento das consequências que o consumo de drogas causa não só no ambiente familiar, mas em toda a comunidade.

\section{MÉTODO}

Trata-se de uma pesquisa descritiva exploratória, de abordagem quantitativa, realizada na Unidade de Referência da Atenção Primária a Saúde $D^{a}$ Claudia Vitorino, na cidade de Rio Branco Acre.

Nos meses de outubro e novembro do ano de 2014 foram realizadas 1.797 consultas de pré-natal na unidade de saúde em estudo e a amostra constituiu-se das que referiam uso de alguma substância química durante o período da gestação e a amostra foi composta pelas 100 usuárias grávidas que informaram que eram usuárias de droga e que aceitaram em participar da pesquisa.

As consequências do uso de drogas durante a gravidez foi a variável independente utilizada e as respostas coletadas dos participantes da pesquisa constituíram o grupo de variáveis dependentes.

O projeto de pesquisa foi aprovado pelo Comitê de Ética e Pesquisa (CEP) do Hospital das Clínicas de Rio Branco Acre com o parecer número 146.856 . Iniciando-se a coletas dos dados após a autorização da instituição onde foi realizada a coleta dos mesmos. As grávidas eram orientadas sobre os de- talhes da pesquisa e, estando de acordo com a participação, o Termo de Consentimento Livre e Esclarecido era assinado pela participante da pesquisa que obedeceu as recomendações da resolução 466 de 2012 do Conselho Nacional de Saúde que regulamenta a realização de pesquisa envolvendo seres humanos.

Os dados foram coletados através de entrevista com as grávidas durante as consultas de pré-natal e as respostas eram registradas em um questionário contendo os dados sociodemograficos e sobre vários tipos de substâncias químicas que eram consumido pelas participantes da pesquisa. Essa coleta de dados ocorreu durante toda a consulta de rotina de pré-natal.

O instrumento de coleta de dados utilizado foi um questionário estruturado com perguntas simples e fechadas, com o objetivo de identificar as substâncias químicas mais consumidas pelas muIheres usuárias.

Os critérios de inclusão adotados foram: Aceitar em participar da pesquisa, ser maior de 18 anos e ser dependente química de algum tipo de droga que poderia ser licita ou ilícita e, os critérios de exclusão adotados foram: Não aceitar em participar da pesquisa, ser menor de 18 anos, não ser dependente química.

\section{RESULTADOS E DISCUSSÃO}

Durante um período de 9 semanas (01 de outubro a 28 de novembro de 2014), foram realizadas 1.797 consultas de pré-natal na Unidade de Referência da Atenção Primária a Saúde Dra Claudia Vitorino no Município de Rio Branco Acre. Essas mulheres tinham idade entre 18 a 37 anos. Identificou-se que no período da gravidez as gestantes continuavam fazendo uso das seguintes drogas: $(47 ; 2,61 \%)$ faziam uso de crack, $(37 ; 2,05 \%)$ consumia bebida alcoólica, $(22 ; 1,22 \%)$ fumava maconha, (19; 1,00\%) eram fumantes de cigarro normal e (17; 0,94\%) faziam uso de cocaína (Tabela 1). 
Tabela 1 - Porcentagem de mulheres grávidas usuárias de drogas atendidas em uma unidade de saúde da atenção primária no município de Rio Branco, Acre, Brasil 2014.

\begin{tabular}{c|cc}
\hline VARIÁVEL & $F(A) \mathbf{N}=100$ & $F(\%)$ \\
\hline Fumantes & 19 & $1,00 \%$ \\
Bebida Alcoólica & 37 & $2,05 \%$ \\
Uso da Maconha & 22 & $1,22 \%$ \\
Uso da cocaína & 17 & $0,94 \%$ \\
Uso do Crack & 47 & $2,61 \%$ \\
\hline
\end{tabular}

Analisando-se, inicialmente os resultados deste estudo realizado em uma comunidade carente do município de Rio Branco Acre, verificou-se que entre as mulheres atendidas que se declararam usuária de alguma substância química (19; 1,00\%) eram fumantes e que não pararam de fumar mesmo durante a gravidez. O hábito de fumar durante a gravidez pode trazer sérios riscos para à saúde da mulher como para a do feto como a placenta previa, episódios de hemorragia materna, abortos espontâneos, nascimentos prematuros, complicações durante o parto, bebês com baixo peso, mortes fetais e de recém-nascidos. O uso do cigarro por uma gestante é capaz de acelerar em poucos minutos, os batimentos cardíacos do feto, devido ao efeito da nicotina sobre o seu aparelho cardiovascular. Além disso, quando a mãe fuma durante a amamentação, a nicotina e absorvida pela a criança através do leite materno.

Para vários autores o hábito de fumar pode causar retardo do crescimento fetal além de aumentar o risco de trabalho de parto pré-termo, restrição de crescimento fetal e baixo peso ao nascer. O tabagismo também aumenta o risco de complicações gestacionais relacionadas à insuficiência vascular, tais como, insuficiência uteroplacentária e deslocamento de placenta. O fumo também pode causar déficit de atenção, transtorno de hiperatividade e problemas de comportamento e de aprendizado, quando a criança atinge a idade escolar.
Verificou-se que o uso do etanol foi a segunda drogas mais consumida pelas grávidas que foram entrevistadas durante a realização desta pesquisa, apresentando uma porcentagem de (37; 2,05\%). O hábito de consumir etanol durante a gravidez se caracteriza como um grave problema de saúde pública em todo o mundo, porque pode causar conseqüências graves tanto para a mãe como para o feto. A síndrome alcoólica fetal é uma das causas mais conhecidas de retardo mental nos Estados Unidos, sendo caracterizada por restrição de crescimento, atraso de desenvolvimento e características faciais distintas.

É importante ressaltar que o cuidado com as gestantes dependente de etanol e de outras drogas é complexo e exige um preparo específico por parte dos enfermeiros. Os profissionais devem estar conscientes das características únicas de cada usuária. O principal obstáculo para o tratamento das mulheres dependentes, em geral, é o preconceito que sofrem por parte da própria comunidade. Nas mulheres grávidas, esse preconceito se multiplica, tornando quase impossível um pedido de ajuda e como conseqüência, essas gestantes raramente fazem acompanhamento pré-natal e, quando fazem, ocultam o uso de drogas. Por outro lado, o período gestacional é um período facilitador de sensibilização ao tratamento, por isso se houver preparo por parte da equipe de saúde, é exatamente nessa fase que se consegue uma abstinência completa e du- 
radoura de todas as drogas, tendo em vista que a maioria das mães não querem prejudicar o bebê..(14)

O uso da maconha entre as mulheres que se declararam usuária de alguma droga atendidas na unidade de saúde em estudo foi de $(22 ; 1,22 \%)$ da amostra. A maconha é a droga ilícita de maior consumo durante a gestação segundo alguns autores, mas, nesse estudo o crack e o álcool apresentaram porcentagens superiores ao uso da maconha. Diante dessa problemática o consumo de crack e etanol pode ter apresentado proporções superiores motivado pelo custo financeiro para ter acesso a droga já que o estudo foi realizado em uma população carente onde o desemprego e a miséria é claramente observado nessa população.

A maconha provavelmente é a droga ilícita de maior uso durante a gestação. Seu princípio ativo é o delta-9-tetra-hydrocannabinol (THC), substância lipossolúvel que atravessa com facilidade a barreira placentária. Há poucas evidências dos efeitos deletérios da maconha sobre o feto e as crianças nascidas de mães usuárias, até mesmo pelo uso concomitante e quase sempre presente de outras substâncias, o que não diminui sua crescente importância Porém, estudos relacionados ao uso dos opiáceos por gestantes, além de escassos, estão entre os mais divergentes, pois, na maioria das vezes, cada autor interpreta os resultados de forma diversa, dificultando a conclusão. Entretanto, muitos concordam que as principais consequências do uso de opiáceos estão nas alterações morfológicas fetais. ${ }^{(15)}$

No que se refere ao uso de cocaína entre as pacientes que foram atendidas nas consultas de pré-natal e que se declararam usuárias, verificou-se uma porcentagem de (17; 0,94\%). Essa droga foi a que apresentou a menor porcentagem de consumo entre os participantes da pesquisa.

Os efeitos pré-natais da metanfetamina sobre o feto ainda não são bem conhecidos, mas a ocorrência de alterações morfológicas e funcionais do sistema nervoso, alterações cardíacas e desenvolvimento intrauterino inadequado já foram demonstrados em alguns estudos. Diversos estudos têm procurado correlações clínicas entre o uso de cocaína antenatal e suas perturbações no desenvolvimento futuro da criança, mas poucos têm focado em doses-efeitos, principalmente no que diz respeito à função neurológica além do período neonatal. Onze estudos relacionaram o uso de cocaína durante a gestação com os efeitos sobre o feto. Três estudos demonstraram que o baixo peso ao nascimento está relacionado com o uso da droga, sendo que revisão sistemática mostrou a correlação entre prematuridade e baixo peso com fatores maternos, ambientais, sociais, nutricionais, genéticos, psicossociais, infecciosos e também ao estilo de vida. ${ }^{(16)}$

Também foi observado entre as gestantes que participaram da pesquisa e se declararam usuárias de alguma droga, que o crack foi a droga que apresentou uma maior porcentagem de consumo com (47; $2,61 \%)$ entre as grávidas que realizaram consultas de acompanhamento do pré-natal nos meses de outubro e novembro de 2014, em uma Unidade de Referência da Atenção Primária a Saúde no município de Rio Branco Acre. Sendo que esse numero é bem maior porque nem todas realizam acompanhamento medico durante a gravidez.

A grande maioria das gestantes dependentes químicas, especialmente do crack, não faz nenhum tipo de acompanhamento pré-natal nem procura orientações médica durante a gravidez, pois sua rotina se faz nas ruas, com o uso de drogas. Elas costumam chegar à maternidade apenas na hora do parto e isso acaba dificultando ainda mais a identificação delas e do número de crianças nascidas nessa situação. Sobre os efeitos do crack nos bebês filhos de mães usuárias, os estudos ainda são poucos e de certa forma contraditórios. Não há uma verdade uniforme. Caracteristicamente esses bebês são pouco responsivos, facilmente irritáveis e difíceis de interagir apresentando altos níveis de ansiedade e de estresse. ${ }^{(17)}$

As consequências mais comuns do uso de drogas durante a gravidez são multifatoriais, podendo causar efeitos irreversíveis tanto na gestante como no feto nessas implicações estão incluídos hiper- 
tensão, taquicardia, hipertermia, malformações congênitas, deslocamento prematuro de placenta, abortamento espontâneo, parto prematuro, morte fetal, crescimento intra-uterino retardado, baixo peso ao nascer, irritabilidade, excitação, tremores, convulsões, microcefalia, retardo mental ou transtornos neurológicos no recém-nascido.

Como o uso de drogas nos dias atuais constitui-se como um problema de saúde pública cabe as autoridades e gestores, criarem parcerias para meIhorar a assistência a população usuária de drogas e principalmente as grávidas que se constitui como um grupo vulnerável de pacientes que sofrem com o problema de dependência química. O Ministério da Saúde tem buscado intervir nas causas e efeitos do consumo de drogas, em conjunto com outras políticas sociais, como, por exemplo, a implementação dos Consultórios na Rua.

\section{CONCLUSÕES}

Os resultados do presente estudo permitiram entender que o uso de drogas durante a gravidez podem trazer consequências tanto para a mãe como para o recém-nascido e o diagnóstico desse problema deve ser feito durante a anamnese nas consultas de planejamento familiar e nas consultas de pré-natal.

Das cinco drogas que foram investigadas nesse estudo o crack foi a que apresentou maior porcentagem de usuárias com uma porcentagem de uso de $(2,61 \%)$ entre as mulheres que se declararam usar alguma droga durante a gravidez e a segunda mais consumida foi o etanol com $(2,05 \%)$ de uso, a terceira foi a maconha com $(1,22 \%)$, a quarta foi o cigarro normal com $(1,00 \%)$ e a quinta foi a cocaína com (0,94\%). Essas porcentagens em números parecem pequena mas em relação a população de estudo representa uma grande quantidade de muIheres que se declararam fazer uso de alguma droga durante a gravidez.

A real extensão dos danos causados pelo o uso de várias drogas durante a gravidez como a maco- nha, o crack, a cocaína ainda hoje é desconhecido, precisando mais estudos nessa área e os a longo prazo ainda são mais difíceis de serem detectados devido aos raros estudos longitudinais sobre o assunto. Outro fator que dificulta os resultados das pesquisas é a associação do uso de várias drogas pelos participantes das pesquisas, isso porque existem a associação de substâncias diferentes utilizadas pelos traficantes no prepara das drogas.

Apesar das conseqüências do uso de drogas serem catastróficos. Eles estão presentes em nossa sociedade e são graves tanto para a mãe como para o feto. Portanto enfatizo a importância da realização de mais pesquisas abordando e discutindo sem preconceito, sem medo, sem repressão e sim como um problema de saúde pública com sérias conseqüências para a sociedade.

Propõem-se a elaboração de políticas públicas e programas assistenciais específicos, mais adequados e direcionados a grávida no que diz respeito à prevenção e tratamento do uso de drogas, visto que estes permitem estabelecer estratégias de prevenção que minimizem os efeitos e as consequências causadas pela droga na gestante e no recém-nascido. Em suma, mais estudos e discussões sobre drogas e gestação, ainda são necessários, sendo este tema merecedor de ser vastamente abordado e discutido entre as diversas profissões da área da saúde.

\section{REFERÊNCIAS}

1. Brasil. Ministério da Saúde. Secretaria de Vigilância em Saúde. Plano emergencial de combate ao uso nocivo de álcool e outras drogas. Brasília, DF; 2O1O. Disponível em:

2. http://bvsms.saude.gov.br/bvs/sus/pdf/junho/ MS_plano_emergencial_combate_uso_alcool_ drogas_04O6.pdf

3. Brasil. Ministério da Saúde. Secretaria Executiva. Coordenação Nacional de DST/ Aids. A política do ministério da saúde para a atenção integral a usuários de álcool e outras drogas. Brasília, DF; 2003. 
4. Mesquita MA, Segre CAM. Freqüência dos efeitos do álcool no feto e padrão de consumo de bebidas alcoólicas pelas gestantes de maternidade pública da cidade de São Paulo. Rev. bras. crescimento desenvolv. Hum. 2009;19(1):63-77.

5. Barbosa T, Miranda MP, Nunes GS, Schutte TS, Santos K, Monteiro DLM. Manifestações do uso de maconha e opiodeos durante a gravidez. Femina. 2011;39(7).

6. Chaves NH, Moreira RAS. Fundamentos de obstetrícia básica. $2^{a}$ ed. São Paulo: Atheneu; 2007.

7. Holztrattner JS. Crack, gestação, parto e puerpério: um estudo bibliográfico sobre a atenção à usuária [monografia]. Porto alegre: Universidade Federal do Rio Grande do Sul; 2010.

8. Stippo MAC, Assis LS, Leite JL, Andrade MP, Cunha NM, Simões RD. O consumo do álcool e as doenças cardiovasculares: uma análise sob o olhar da enfermagem. Esc. Anna Nery Rev. Enferm. 2007;11(4):581-85.

9. Pinho PH et al. Reabilitação psicossocial na atenção aos transtornos associados ao consumo de álcool e outras drogas: a concepção de profissionais de saúde. Rev. Esc. Enferm. USP. 2009;43(Esp. 2):1261-6.

10. Brasil. Ministério da Saúde. Secretaria de Atenção à Saúde. Departamento de Ações Programáticas Estratégicas. Gestação de alto risco: manual técnico. $5^{\mathrm{a}}$ ed. - Brasília, DF; 2010.

11. Souza J, Kantorski LP. Embasamento político das concepções e práticas referentes às drogas no Brasil. SMAD, Rev. eletrônica saúde mental alcool drog. 2OO7;3(2).

12. Oliveira EB, Bittencourt LP, Carmo A. A importância da família na prevenção do uso de drogas entre crianças e adolescentes: papel materno. SMAD, Rev. eletrônica saúde mental alcool drog. 2008;4(2).

13. Aliane PP. Uso de álcool na gestação e sua relação com sintomas depressivos no pós-parto [dissertação]. [ Ribeirão Preto]: Faculdade de Medicina de Ribeirão Preto (USP); 2008.

14. Leveno KJ. Manual de obstetrícia de Williams. 21a ed. Porto Alegre: Artmed; 2005.

15. Maia JA, Mesquita RO. Experiências e percepções de mães usuárias de drogas atendidas em uma unidade de saúde da atenção primária. Ariquemes (RO). Revista Científica da Faculdade de Educação e Meio Ambiente. 2015;6(1):1-13.

16. Barbosa TD, Miranda MP, Nunes GF, Schutte TS, Santos K, Monteiro DLM. Manifestações do uso de maconha e opiáceos durante a gravidez. Femina. 2011; 39(8).

17. Cembranelli E, Campos LRF, Portella M, Abreu PVC, Salomão PC, Monteiro DLM. Consequências do uso de cocaína e metanfetamina durante a gravidez. Femina. 2O12; 4O(5).

18. Camargo PO, Martin MFD. Os efeitos do crack na gestação e nos bebês nascidos de mães usuárias: Uma revisão bibliográfica. Cad. Ter. Ocup. 2014;22(Supl. esp.):161-169. 